\title{
Introduction to cognitive artificial systems
}

\section{Antonio Lieto \& Marco Cruciani}

To cite this article: Antonio Lieto \& Marco Cruciani (2015) Introduction to cognitive artificial systems, Connection Science, 27:2, 103-104, DOI: 10.1080/09540091.2015.1007700

To link to this article: https://doi.org/10.1080/09540091.2015.1007700

曲 Published online: 06 May 2015.

Submit your article to this journal 중

III Article views: 470

View Crossmark data $\nearrow$

4 Citing articles: 1 View citing articles 준 


\title{
INTRODUCTION
}

\section{Introduction to cognitive artificial systems}

\author{
Antonio Lieto* and Marco Cruciani
}

The research in Artificial Intelligence (AI) has been based, from an historical standpoint, on a strong collaboration between computer scientists, psychologists, engineers, philosophers, linguists and biologists working in the Cognitive Science field. This collaboration, fostered by the influence of the cybernetic approach to the study of natural and artificial systems (Wiener, 1965), has produced - along the years - the development of fruitful research lines in bionics, robotics, biologically and neurally inspired systems and, more in general, in the area of cognitive artificial systems and systems science.

After decades of mutual and pioneeristic collaborations, however, Artificial Intelligence and Cognitive Science have produced several sub-disciplines, each with its own goals, methods and criteria for evaluation. This fragmentation, on one hand, has facilitated the development of some AI systems able to produce super-human competences, but only in very restricted domains (such as in theorem proving, or in games such as chess, Jeopardy, etc.). On the other hand, however, it has been based on a divide et impera approach that has significantly inhibited the cross-field collaborations and the scientific efforts aimed at investigating a more general picture of what natural and artificial intelligence are, and how intelligent artefacts can be designed by taking into account the insights coming from the natural world.

In more recent years, however, the area of cognitively inspired artificial systems has attracted a renewed attention both from academia and industry (Besold, d'Avila Garcez, Kühnberger, \& Stewart, 2014) and the awareness about the need for additional research in this interdisciplinary field has gained widespread acceptance. The gap between natural and artificial intelligence, in fact, is nowadays still enormous (Sloman, 2014) and the research in this area seems now crucial for the development of artificial systems endowed with human-level intelligence.

The special issue collects a selection of revised and extended versions of the papers presented at the first International Workshop on Artificial Intelligence and Cognition (AIC13), held in Turin, Italy, on the 3rd of December 2013 (Lieto \& Cruciani, 2013). The papers have been selected by the editors after a double round of peer reviews, and discuss some possible solutions to well-known problems in the area of cognitively inspired Artificial Intelligence. The problems considered span from the issue regarding the cognitive control of robotic artifacts to that ones dealing with the aspects of intelligent associative learning, common sense reasoning and neuropsychological modelling of the artificial cognition. In particular, the paper "Using a cognitive architecture for general purpose service robot control", by Jordi-Ysard Puigbo, Albert Pumarola, Cecilio Angulo and Ricardo Tellez, presents a humanoid service robot equipped with a set of simple action skills including navigating, grasping and recognising objects or people.

*Corresponding author Email: lieto.antonio@gmail.com 
The robot is able to complete a voice command in natural language that encodes a complex task and decide which of its skills should be activated (and in which sequence) by using the SOAR cognitive architecture. The paper "Discriminating and Simulating Actions with the Associative Self-Organizing Maps", by Miriam Buonamente, Haris Dindo and Magnus Johnsson, presents a method based on the Associative Self Organizing Map (A-SOM) used for learning and recognising actions. The authors show how their A-SOM based systems, once learnt to recognise actions, use this learning to predict the continuation of an observed initial movement of an agent, anticipating, in this way, its intentions. The paper "A Knowledge-Based System for Prototypical Reasoning", by Antonio Lieto, Andrea Minieri, Alberto Piana and Daniele P. Radicioni, presents a knowledge-based system equipped with a hybrid, cognitively inspired, architecture. Such a system, tested in a Question-Answering setting, extends the representational and reasoning capabilities of classical ontology-based systems towards the scenarios envisioned by the prototype theory. Finally, the paper "Approaching Neuropsychological Tasks through Adaptive Neurorobots", by Onofrio Gigliotta, Paolo Bartolomeo and Orazio Miglino, presents a study where an artificial embodied neural agent, equipped with a pan/tilt camera and provided with different neural and motor capabilities, deals with a well-known neuropsychological test: the cancellation task. The paper shows that embodied agents provided with additional motor capabilities outperform simple pan/tilt agents even when controlled by more complex neural controller, thus pointing out that the sole neural computational power cannot explain the (artificial) cognition which emerged throughout adaptive processes.

We would like to dedicate this special issue to the memory of Leonardo Lesmo, which strongly supported the idea of organising the AIC series of workshops and would like to thank Amanda Sharkey, Tony Prescott and the editorial team of Connection Science for their precious support.

Torino and Trento, January 2015

\section{References}

Besold, T. R., d'Avila Garcez, A., Kühnberger, K.-U., \& Stewart, T. C. (Eds.). (2014). Neural-symbolic networks for cognitive capacities (Special issue). Biologically Inspired Cognitive Architectures, 9, 1-122.

Lieto, A., \& Cruciani, M. (Eds.). (2013). Artificial Intelligence and Cognition. Proceedings of the first international workshop on artificial intelligence and cognition (AIC 2013) (vol. 1100, pp. 1-171), CEUR-ws, Aachen, Germany.

Sloman, A. (2014). How can we reduce the gulf between artificial and natural intelligence? Proceedings of the 2 nd international workshop on artificial intelligence and cognition (AIC 2014) (vol. 1315, pp. 1-13), Lieto Antonio, Radicioni, D. P., \& Cruciani, M. (Eds.), CEUR, Aachen, Germany.

Wiener, N. (1965). Cybernetics or control and communication in the animal and the machine, Vol. 25, Cambridge, MA: MIT Press. 